\title{
Políticas e Sistemas de Saúde Ibero-americanos - uma análise comparada: Entrevista de Eleonor Minho Conill. ${ }^{1}$
}

\section{Policies and Health Systems Ibero-American - a comparative analysis.}

\section{Políticas y Sistemas de Salud Iberoamericanos - un análisis comparado.}

Tamires Marinho dos SANTOS ${ }^{2}$

Rackynelly Alves Sarmento SOARES ${ }^{3}$

Ana Valéria Machado MENDONÇA ${ }^{4}$

O que é o OIAPSS? Como ele surgiu? Qual sua estrutura, missão, objetivos?

CONILL - A criação do Observatório Ibero-Americano de Políticas e Sistemas de Saúde (OIAPSS) ocorreu em abril de 2011, em um seminário organizado em Mérida, Espanha, por iniciativa do Conselho Nacional de Secretarias Municipais de Saúde (CONASEMS) e da Federación de lasAsociaciones em Defensa de La Sanidad Pública (FADSP). O seminário contou com a presença de representantes de instituições governamentais e não-governamentais tais como, o Ministério da 1 Eleonor Minho Conill é graduada em Medicina pela Universidade Federal do Rio Grande do Sul, Mestre em Saúde Comunitária pela Université de Montréal e Doutora em Desenvolvimento Econômico e Social pela Université Paris I (Pantheon -Sorbonne). Atuou como docente e pesquisadora nas Universidades Federais do Rio Grande do Sul, Rio de Janeiro, Santa Catarina e na Escola Nacional de Saúde Pública da Fundação Oswaldo Cruz. Trabalha na área de políticas, planejamento, organização, avaliação e análise comparada de sistemas de saúde. Foi consultora de pesquisa na Escola de Governo da FIOCRUZ e na Red de Investigaciónen Sistemas y Servicios de SaluddelConoSur. Integra a Comissão de Política Editorial da Revista Ciência e Saúde Coletiva da ABRASCO e, é membro do corpo editorial da Revista Saúde em Debate do CEBES. Colaborou na fundação do Observatório Ibero-Americano de Políticas e Sistemas de Saúde (OIAPSS) coordenando o projeto para desenvolvimento da matriz analítica deste Observatório. 2 Assessora de Comunicação do Núcleo de Estudos em Saúde Pública da Universidade de Brasília (NESP/UnB) tamiresmarinhotm@gmail.com - SCLN 406 Bloco A $2^{\circ}$ andar - Asa Norte - Brasília/DF - CEP: 70847-510 3 Pesquisadora do em Saúde Pública da Universidade de Brasília (NESP/UnB). Doutorando do Programa de Pós-graduação em Modelos de Decisão e Saúde, Universidade Federal da Paraíba, João Pessoa-PB, Brasil. E-mail: rackynelly@unb.br

4 Professora do Programa de PósGraduaçãoemSaúdeColetiva da Universidade de Brasília. Coordenadora do Núcleo de EstudosemSaúdePública da UnB.E-mail: valeriamendonca@gmail.com 
Saúde do Brasil, o Conselho Nacional de Secretários de Saúde (CONASS), o Centro Brasileiro de Estudos de Saúde (CEBES), a Escola Nacional de Saúde Pública (ENSP/FIOCRUZ), a Associação Brasileira de Saúde Coletiva (ABRASCO), a UniversidadJaveriana da Colômbia, a Universidad Nacional de Rosário, Argentina, a Consejería de Sanidad y Dependencia de Junta de Extremadurae a Fundación $1^{\mathbf{0}}$ de Mayo da Espanha, entre outros.

Essa Fundação financiou uma publicação que compila os trabalhos apresentados nesse primeiro seminário e, que está disponível em nosso site (www.oiapss.org) juntamente com o que denominamos Documento Base. Este documento aprofunda a história e os fundamentos que orientam o OIAPSS.

Os objetivos do Observatório estão focados em proporcionar um espaço de comunicação e intercâmbio para contribuir na qualidade dos sistemas públicos de saúde. Ou seja, facilitar a criação de uma rede de instituições para acompanhamento e discussão da situação e das tendências desses sistemas e das políticas de saúde.

Um Observatório se constitui num centro de informação, monitoramento, análise e avaliação, serviços de assessoria, apoio a gestão, difusão, comunicação e transferência de conhecimento. A observação é diferente da investigação, pois seu objetivo principal é agregar valor ao conhecimento ao facilitar sua utilização. A abundância de informações que se encontram, em geral dispersas e pouco sistematizadas, torna necessários instrumentos para disponibilizar sínteses de questões estratégicas para a gestão e para os movimentos sociais.

A comparação tem se constituído num recurso importante para identificar tendências de blocos regionais ou intervenções para melhorar a qualidade dos serviços. Portugal e Espanha acumularam um conhecimento interessante na condução de sistemas nacionais com ênfase na atenção primária /APS, mas as reformas dos sistemas de países anglo-saxões tiveram maior influência na América Latina, havendo pouca interação com esses países. O intercâmbio entre os países latino-americanos, embora essencial para uma perspectiva internacional comparada, é ainda restrito. Mas além de comparar indicadores ou estabelecer padrões, a perspectiva comparada adotada no OIAPSS enfoca a análise de temáticas e desafios comuns no contexto da crescente internacionalização das relações sociais e suas repercussões no âmbito da saúde.

Na Oficina realizada na Espanha no momento de criação do Observatório foram definidas as seguintes linhas de trabalho:

- Financiamento/Alocação;

- Relação público/privado/ Modelos de gestão público/privado;

- Descentralização/Regionalização/Redes de Atenção à Saúde; 
- Atenção Primária da Saúde-APS;

- Gestão Clínica;

- Monitoramento e Avaliação;

- Medicalização/influência da indústria farmacêutica;

O OIAPSS está sediado no Núcleo de Estudos em Saúde Pública da Universidade de Brasília (NESP/UnB) que, em parceria com o CONASEMS e com a Secretaria de Gestão Estratégica e Participativa do Ministério da Saúde (SGEP/MS), tem garantido a continuidade desta iniciativa sob coordenação geral do Dr. Sílvio Fernandes, que esteve responsável pelas relações internacionais do CONASEMSdurante muitos anos. Foram criados Núcleos do OIAPSS ou garantidas parcerias em vários países como: Argentina, Espanha, Colômbia, Chile, México, Peru, Uruguai, Paraguai, Portugal, Venezuela e Honduras. Há um grupo operativo que acompanha as atividades de modo a prestar contas junto ao CONASEMS e ao MS, instituições que tem assegurado nosso financiamento até agora.

\section{A REDE DE PESQUISADORES}

SANTOS, SOARES, MENDONÇA - Como foi articulada a rede de pesquisadores do OIAPSS?

CONILL - A rede de pesquisadores se aglutinou em torno de um projeto concreto que foi o desenvolvimento de matriz analítica para acompanhamento dos sistemas de saúde de países iberoamericanos participantes do Observatório. Este projeto iniciou em julho de 2011 e, coincide praticamente com o início do OIAPSS. Visava responder a seguinte pergunta: O que deve ser observado num sistema de saúde? E, como fazer isso? Ou seja, teve como objetivo desenvolver uma tecnologia de informação que permitisse acompanhar os sistemas de saúde e suas tendências.

SANTOS, SOARES, MENDONÇA - Atualmente ele conta com a colaboração de quantos países/membros?

CONILL - No caso da matriz, houve a participação de sete países provenientes dos primeiros Núcleos do Observatório e, que são: Argentina, Brasil, Colômbia, Espanha, Paraguai, Peru e Portugal. As temáticas foram divididas conforme as expertises técnicas dos pesquisadores indicados pelos Núcleos. Além disso, contamos com a colaboração de consultores de várias Universidades e instituições brasileiras, principalmente do Laboratório de Informação em Saúde do Instituto de Informação Científica e Tecnológica da Fundação Oswaldo Cruz (LIS/ICICT/ FIOCRUZ,) cuja assessoria foi essencial para o projeto, uma vez que nos inspiramos num trabalho já realizado por esse Laboratório, o Projeto "Desenvolvimento de metodologia de avaliação do desempenho do sistema de saúde" (PRO-ADESS). Também tivemos ajuda do Observatório de Saúde da Região 
Metropolitana de São Paulo, do Instituto de Direito Sanitário (IDISA), do Instituto de Saúde Coletiva da UFBA, da Escola de Saúde Pública da USP, da UFSC, entre outros.

Mas, enquanto Observatório a rede de parcerias tem se ampliado, incluindo a participação de outros países como Chile, México, Uruguai, Venezuela e Honduras. Alguns desses países, já demonstraram interesse em se incorporar ao projeto Matriz.

SANTOS, SOARES, MENDONÇA - Sabemos que a articulação de uma rede internacional requer muito esforço e comprometimento dos atores envolvidos. No caso do OIAPSS, quais são os obstáculos enfrentados pela rede de pesquisadores?

CONILL - Tivemos algumas dificuldades determinadas pela distância geográfica, pela irregularidade do financiamento, diferença de períodos de trabalho entre países europeus e latinoamericanos. Janeiro e fevereiro são meses de férias no Brasil, mas de intensa atividade em Portugal e Espanha, por exemplo. Ao contrário, em julho e agosto trabalhamos e eles estão em recesso.

\section{MATRIZ ANALÍTICA}

SANTOS, SOARES, MENDONÇA - Numa perspectiva comparada, o projeto da Matriz Analítica, coordenado por você ao longo de mais de quatro anos, reuniu dados de sete países com aspectos semelhantes, porém, ao mesmo tempo, bastante distintos. Nesse sentido, quais foram os principais desafios no desenvolvimento da pesquisa e qual a importância desse trabalho no contexto dos sistemas de saúde dos países ibero-americanos?

CONILL - Em primeiro lugar, tivemos um ganho científico e tecnológico importante ao desenvolver um instrumento para gestão da informação que permite o acompanhamento integrado de séries temporais de sessenta e cinco (65) indicadores. O modelo de programação web desenvolvido garante o livre acesso a esse conjunto comparativo de informações e de séries históricas na plataforma virtual do OIAPSS e, que poderá ser adaptado conforme novas necessidades ou temáticas específicas dos países. Ou seja, não é um modelo estático nem apenas um relatório de pesquisa, é uma tecnologia de informação. Mas, para um melhor entendimento do que representa o ganho ao qual me refiro, é conveniente citar o modo como está estruturada a matriz.

Os indicadores selecionados estão distribuídos em três grandes blocos temáticos, com as seguintes dimensões e subdimensões:

1- Determinantes - demográficos (estrutura demográfica, razão de dependência), socioeconômicos (renda, trabalho, desigualdade, educação), condições de vida (nutrição, saneamento, violência, saúde mental, mobilidade urbana);

2- $\quad$ Construção social da política de saúde (legislação de cada país, segundo princípios organizativos de um sistema universal sob controle público); 
3- Condicionantes- complexo produtivo (desenvolvimento e inovação, medicalização, incorporação tecnológica, balança comercial), financiamento (gasto setorial, composição público-privado); trabalho em APS (recursos humanos);

4- Desempenho: acesso (cobertura, oferta de recursos), efetividade (mortalidade e morbidade evitável, intervenções e programas marcadores), adequação (procedimentos não esperados).

Essas dimensões, subdimensões e indicadores sugeridos pelos pesquisadores passaram por um processo de validação e, por uma coleta exploratória com ajustes posteriores para garantir a viabilidade e sustentabilidade da matriz, em função dos sistemas de informações existentes.

Também trabalhamos com indicadores que trazem novidades no acompanhamento desses sistemas, pois ao tratar do complexo produtivo da saúde podemos detectar tendências importantes, como é o caso da balança comercial na área da assistência farmacêutica. Este é um importante condicionante dos sistemas de serviços que, em geral, não é acompanhado pelos organismos internacionais. Nos determinantes, utilizamos indicadores que abrangem subdimensões como nutrição, violência, saúde mental e mobilidade urbana. E na educação trabalhamos com os dados do Programme for InternationalStudentAssessment(PISA) da UNESCO que permitem acompanhar a qualidade da situação educacional em cada país.

No entanto, trabalhar com países de um bloco geo-político e cultural cujos sistemas de saúde não haviam sido objeto de estudos comparados, trouxe desafios adicionais a complexidade já inerente a este tipo de pesquisa. Portugal e Espanha integram os bancos de dados da Organization for EconomicCo-operationandDevelopment /OECD, enquanto os demais países da América Latina participam dos Indicadores e Dados Básicos da América.

Há um empenho técnico no tratamento das informações dessas bases com limpezas anuais de indicadores com problemas de validade e acréscimo de outros por novas necessidades. No caso da América Latina, além da padronização dos dados de mortalidade que permite comparações, são também feitas correções através de estimativas de subregistro e de causas mal definidas. Mas sabemos que ainda existem muitas diferenças na qualidade dos sistemas de informações.

SANTOS, SOARES, MENDONÇA - A matriz analitica foi o primeiro grande produto do OIAPSS, e agora, partindo desse ponto, o que podemos esperar desse observatório? Que surpresas o OIAPSS nos reserva?

CONILL - A análise dos indicadores da matriz e seus resultados devem ser discutidos com o auxílio de bibliografia complementar, estudos qualitativos e análises conjunturais que possam contextualizar as informações quantitativas em cada país. Mas acho interessante assinalar alguns pontos. Por exemplo, na área temática que trata dos determinantes temos dados que mostram que, embora haja uma tendência de diminuição, a mortalidade por homicídios permanece muito alta, 
principalmente na Colômbia e no Brasil, neste caso 27.5 e 28 vezes mais elevada do que em Portugal e Espanha, conforme dados do último ano disponível na série histórica coletada (2010). No entanto, em Portugal observa-se um aumento da mortalidade por suicídio e na Espanha o crescimento do índice de Gini. Estes indicadores podem alertar para efeitos da crise econômica e seus reflexos na saúde, sendo importante acompanhá-los. Também, nota-se que o sobrepeso está em ascensão em todos os países com todas as implicações que isto representa para a prevalência e complicações das doenças crônicas.

$\mathrm{Na}$ área dos condicionantes os indicadores alertam para um sério desequilíbrio na balança comercial brasileira em função da importação crescente de medicamentos. Já no que se refere à área de desempenho, nota-se que temos avançado na saúde materna e infantil, por exemplo, com uma diminuição importante da mortalidade por doenças respiratórias agudas em crianças menores de 5 anos. Mas necessitamos melhorar com relação à mortalidade por doenças cerebrovasculares, cardiopatia isquêmica e diabetes.

Convido a todos a conhecerem a Matriz de Indicadores no site do OIAPSS e, o Relatório Final que expõe esses resultados com detalhes. Também é interessante ler sobre as etapas do percurso metodológico e, consultar a matriz conceitual onde apresentamos uma revisão bibliográfica fundamentando os conceitos utilizados e as escolhas das dimensões, subdimensões e indicadores.

SANTOS, SOARES, MENDONÇA - Quais os principais pontos fortes e as fragilidades (limitações) que podemos destacar na Matriz do OIAPSS?

CONILL - Como já mencionei, desenvolvemos uma tecnologia de informação que permite um panorama integrado dos sistemas. Num levantamento que realizamos ao iniciar o OIAPSS sobre os tipos de Observatórios na área da saúde identificamos cem portais, dos quais trinta pertenciam a América Latina e ao Caribe. No entanto, a maior parte deles se concentrava no acompanhamento de determinadas temáticas ou de grupos específicos da população. Um ponto forte da matriz desenvolvida é que propõe uma abordagem integrada das políticas e dos sistemas de saúde. Outro aspecto a destacar é que trabalhamos com séries temporais que permitem acompanhar tendências, o que é fundamental para a formulação e o seguimento de políticas informadas por evidências. Também, o fato de termos uma tecnologia com programação web que permite um desenvolvimento contínuo e está facilmente a disposição de todos.

A análise dos resultados do desenvolvimento da matriz mostra que a área temática dos determinantes sociais é aquela com maior grau de completitude de indicadores. Nas outras, foi necessário modificar e adaptar alguns dos indicadores previstos devido à dificuldade nas fontes de dados, principalmente em áreas temáticas e dimensões que propunham enfoques inovadores, (complexo produtivo da saúde, financiamento, atenção primária).

Embora a APS tenha sido selecionada como uma das dimensões que condicionam o desempenho dos sistemas de saúde devendo ser acompanhada, não foi possível obter dados que viabilizassem 
uma comparação entre os países ibero-americanos. Os recursos humanos foram considerados um marcador importante da implementação dessa política, mas será necessário um desdobramento específico do estudo a fim de aprimorar esta importante dimensão analítica (uniformização de conceitos, fontes para coleta de dados secundários). Essa tarefa deveria ser objeto de maior atenção daqueles que organizam os atuais sistemas de informações. É surpreendente que num tema enfocado por organismos internacionais, objeto de políticas governamentais e de inúmeros estudos acadêmicos durante várias décadas, ainda permaneçam questões conceituais e operacionais que dificultam seu acompanhamento de um modo objetivo.

A contribuição central deste projeto foi o desenvolvimento de uma tecnologia de informação que, por isso mesmo, necessita continuidade e aperfeiçoamento. Este é nosso "calcanhar de Aquiles", sua principal fragilidade. Ou seja, seguimos com o grande desafio: a informação só é útil se a sociedade e os formuladores de política, de fato, a levarem em consideração em suas demandas e decisões. Fizemos uma parte da tarefa ao disponibilizar a matriz, mas entender sua utilidade e aproveitá-la é um esforço de todos. 